EPJ Web of Conferences 32, 03005 (2012)

DOI: 10.1051/epjconf/20123203005

(C) Owned by the authors, published by EDP Sciences, 2012

\title{
Conceptual design of ITER ECE receiver systems and their performance parameters
}

\author{
Hitesh Kumar B. Pandya ${ }^{1}$, V.S. Udintsev², G. Vayakis ${ }^{2}$ and Max Austin ${ }^{3}$ \\ ${ }^{1}$ ITER-India, Institute for Plasma Research, Bhat, Gandhinagar-382 428, India \\ ${ }^{2}$ ITER Organization, Cadarache, 13115 St Paul-lez-Durance, France \\ ${ }^{3}$ Fusion Research Center, the University of Texas at Austin, Austin, TX, USA
}

\begin{abstract}
The Electron Cyclotron Emission diagnostic on ITER requires electron temperature profile with $10 \mu \mathrm{sec}$ time and $6-7 \mathrm{~cm}$ spatial resolution. The diagnostic is also useful to study many plasma physics phenomenon like temperature fluctuation, non-thermal electrons population and the power loss due to ECE. The conceptual design being considered for ITER ECE consist of a radiometer of frequency range 122 to 220 $\mathrm{GHz}$ for $\mathrm{O}$-mode radiation measurement and two Fourier transform spectrometers of frequency range 70 to $1000 \mathrm{GHz}$ as measuring instruments for $\mathrm{O}$-mode and $\mathrm{X}$-mode radiation measurement. There are two lines of sight for the collection of cyclotron radiation. One is radial view and other is oblique view at 10 degree [1]. The radiation collected by both view will be transmitted through the polarization splitter boxes and four transmission lines to the diagnostic area. In this paper we will summarize the conceptual design details and changes proposed at the Conceptual Design Review. The required calibration time for the ECE measuring instrument will be discussed in the paper.
\end{abstract}

\section{Introduction}

The Electron Cyclotron Emission (ECE) is measured to determine electron temperature information of the tokamak plasma. This ECE measurement diagnostic method is proposed for ITER plasma parameter measurement. In this the ITER plasma electron temperature radial profile is going to be measured with time resolution of $10 \mu \mathrm{sec}$ and spatial resolution of $6-7 \mathrm{~cm}$. The diagnostic is also going to be used to study for many plasma physics phenomenon. In this the temporal resolution of the ECE system would be utilized for high frequency instability (MHD, NTMs, AEs, turbulence) study. While the wide band $(70-1000 \mathrm{GHz}$ i.e. number of harmonic) measurement of the ECE radiation is useful to study non-thermal electron population and estimate the power loss due to cyclotron radiation[2].

In this paper, we described the conceptual design being considered for ITER ECE receiver systems that includes a radiometer of frequency range 122 to $220 \mathrm{GHz}$ for first harmonic ordinary mode cyclotron radiation measurement and two Fourier transform spectrometers of frequency range 70 to $1000 \mathrm{GHz}$ as measuring instruments for $\mathrm{O}$-mode and $\mathrm{X}$-mode radiation measurement. The total

This is an Open Access article distributed under the terms of the Creative Commons Attribution License 2.0, which permits unrestricted use, distribution, and reproduction in any medium, provided the original work is properly cited. 
transmission loss from front end radiation collector to the receiver detector is estimated and used to calculate required calibration time for the ECE measuring instrument.

\section{Conceptual design of radiometer}

In a superheterodyne radiometer the RF signal is mixed (at a nonlinear device mixer) with a localoscillator signal having larger amplitude compared to the incoming signal (Figure 1). The mixer translates original signal to a much lower frequency called the intermediate frequency (IF). If the carrier signal is $f_{c}$ and the oscillator signal is $f_{o}$ the mixer produces signal at IF frequency $f_{I F}=f_{o} \pm f_{c}$ and in general at many harmonic frequencies $\mathrm{nf}_{\mathrm{o}} \pm \mathrm{mf}_{\mathrm{c}}$ whre $\mathrm{n}$ and $\mathrm{m}$ are integers. The resulting IF frequency signal is amplified to a suitable power level then detected and finally processed for the intended output application.

The proposed O-mode radiometer of frequency range $122-230 \mathrm{GHz}$ is multi-band in the millimeter and sub-millimeter wave frequency range [2]. Each band has one or more than one down conversion system and filter bank as shown in figure 1 . The parameters of the $\mathrm{O}$-mode radiometer are described in Table 1. There is a quasi-optical box for catering the O-mode radiation to the various down convertor systems. The quasi-optical box contains non-polarizing splitters and reflectors.

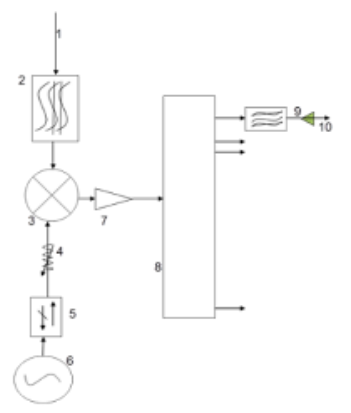
1) RF INPUT $122-139 \mathrm{GHZ}$
2) $121 \mathrm{GHz}$ high pass filter
3) Balanced Mixer of IF $1-18 \mathrm{GHz}$
4) Variable attenuator
5) Isolator
6) Local oscillator of $121 \mathrm{GHZ}$
7) IF Amplifiers
8) Power divider of 18 channels
9) Band pass filter
10) Diode detector

Fig. 1. Schematic block diagram of F-Band Radiometer $122-139 \mathrm{GHz}$

Table 1. O-mode radiometer parameters

\begin{tabular}{|c|c|c|c|c|}
\hline Parameters & \multicolumn{4}{|c|}{$\begin{array}{l}\text { Radiometer with frequency range } \\
122-230 \mathrm{GHz}\end{array}$} \\
\hline Frequency Band & F-Band & D-Band & G-Band & $\begin{array}{l}\text { Millimeter } \\
\text { wave }\end{array}$ \\
\hline Frequency range $(\mathrm{GHz})$ & $122-139$ & $141-169$ & $172-218$ & $222-230$ \\
\hline Number of channels & 18 & 15 & 16 & 3 \\
\hline $\begin{array}{l}\text { Channels separation } \\
(\mathrm{GHz})\end{array}$ & 1 & 2 & 3 & 4 \\
\hline $\begin{array}{l}\text { Transmission line loss } \\
\text { (dB) }\end{array}$ & -13 & -13 & -13 & -13 \\
\hline Total loss up to detector & -44 & -46.3 & -49.5 & -32.3 \\
\hline Two stage IF amplifiers & 80 & 80 & 80 & 70 \\
\hline Total gain & 36 & 33.7 & 30.5 & 37.7 \\
\hline $\begin{array}{l}\text { The sensitivity of the } \\
\text { radiometer for detector } \\
\text { sensitivity of } 1000 \mathrm{~V} / \mathrm{W}\end{array}$ & $4 \times 10^{6}$ & $2.3 \times 10^{6}$ & $1.1 \times 10^{6}$ & $5.9 \times 10^{6}$ \\
\hline Noise temperature $(\mathrm{eV})$ & 8.3 & 9.5 & 10.7 & 8.3 \\
\hline Noise power (W) & $\begin{array}{l}5.9 \times 10^{-10} \text { for } \\
B_{\text {if }}=0.5 \mathrm{GHz}\end{array}$ & $\begin{array}{l}1.4 \times 10^{-10} \text { for } \\
B_{\text {if }}=1 \mathrm{GHz}\end{array}$ & $\begin{array}{l}2.4 \times 10^{-9} \text { for } \\
\mathrm{B}_{\text {if }}=1.5 \mathrm{GHz}\end{array}$ & $\begin{array}{l}2.4 \times 10^{-9} \text { for } \\
B_{\text {if }}=2 \mathrm{GHz}\end{array}$ \\
\hline
\end{tabular}




\section{Conceptual design of Michelson interferometer}

For fusion plasma, for ECE measurement, a polarized interferometer is used. In this, the basic design of the optics is a Martin-Puplett type [3]. The division of the incident wave is attained by a polarizer beam splitter made up of arrays of parallel metal wires. An interferogram is generated by combining two beams at the beam splitter after reflection one beam from a fixed and other beam from a moving mirror. The spectral intensity is obtained by Fourier transformation of the interferogram. Now a day, the path difference is varied by using moving mirror.

\subsection{State-of-the-art in the ITER Michelson interferometer design}

There are three important features for the design driver of the Michelson interferometer for the ITER ECE spectrum measurement. One is the rapid scanning mechanism that decides time resolution of full spectrum measurement. Second is the atmosphere shielding to avoid water vapor absorption of the millimeter wave radiation. Other is throughput of the interferometer. Absolute calibration of the Michelson interferometer by the hot/cold black body source technique depends on the throughput of the interferometer. The interferometer with high throughput can be absolutely calibrated within moderate time scale. These three features need to be optimized in the design of the ITER ECE Michelson interferometer.

The future design of the ITER ECE Michelson will give improvement in the state of the art in ECE spectrum measurement method. The use of all reflection optics for coupling between waveguide transmission line and the interferometer give good throughput. The vacuum tight modular design and an in-vacuum rapid scanning mechanism are suitable for the ITER ECE measurements up to $1 \mathrm{THz}$. For theses design features the Michelson interferometer specifications are proposed in Table 2.

Table 2 Required specifications of the Michelson interferometer

\begin{tabular}{|l|l|}
\hline Spectral range $(\mathrm{GHz})$ & $70-1000$ \\
\hline Frequency resolution $\mathrm{GHz}$ & $7.5-10$ \\
\hline Linear path scan $(\mathrm{mm})$ & $30-40$ \\
\hline Path difference sampling $(\mu \mathrm{m})$ & $50-100$ \\
\hline Scanning repetition rate & $10-20 \mathrm{~ms}$ \\
\hline Instruments throughput $>$ & $0.4 \times 10-4 \mathrm{~m}^{2} \mathrm{Sr}$ \\
\hline Detector System Optical Responsivity $>$ & $2,000 \mathrm{~V} / \mathrm{Watt}$ \\
\hline Detector System Optical N.E.P $<$ & $1.25 \times 10^{-12} \mathrm{Watts}_{\mathrm{Hz}}^{-1 / 2}$ \\
\hline Detector dynamic range $>$ & $29 \mathrm{~dB}$ \\
\hline Number of Michelson interferometer & 2 \\
\hline Duty cycle & Continuous \\
\hline
\end{tabular}

\subsection{Coupling optics and Michelson optics layout}

A circular smooth walled waveguide of $72 \mathrm{~mm}$ diameter is proposed for ITER ECE transmission line for both radial and oblique views following the outcome of the recent Conceptual Design Review for this diagnostic. Two transmission lines (i.e. one for radial and other for oblique measurements of Omode) are kept near input of the $\mathrm{O}$-mode Michelson interferometer and same arrangement is also for $\mathrm{X}$-mode Michelson interferometer input. There are coupling optics placed between the transmission line waveguide and the input of the Michelson interferometer. For optimum coupling between waveguide to optical mirror, there is relation between dimensions of the waveguide (i.e. radius a or longer side a) to the beam waist radius (w) at the input of the waveguide. We used this relation for dimension calculation of the optical components. For smooth-walled circular waveguide optimum 
coupling of $87 \%$, the ratio of the beam waist radius (w) to the radius of the waveguide is 0.76 [4]. Therefore the beam waist radius $(\mathrm{w})$ is $27.36 \mathrm{~mm}$ for the ITER ECE proposed waveguide transmission line.

The coupling optics and the Michelson interferometer optical layout are shown in figure 2. It consists of two Gaussian beam telescope constructed by using four ellipsoidal mirrors. First three mirrors have same focal length $(\sim 33 \mathrm{~cm})$ with diameter of $\sim 11 \mathrm{~cm}$ and last mirror has a focal length $12 \mathrm{~cm}$ with same diameter. The radiation beam size from the corrugated waveguide output to the detector input is calculated by using Gaussian beam optics theory and shown in figure 2 . This size is useful to decide the size of the optical components. The size of the optical components should be more than 4 times the radius of beam for optimum coupling $(\sim 99 \%)$.

Other alternate for transmission line waveguide is also proposed. In this, there are two types of waveguide at the input of the interferometer. One is circular corrugated waveguide of diameter 63.5 $\mathrm{mm}$ and other is rectangular smooth walled waveguide of dimension $72 \times 34 \mathrm{~mm} 2$. Above same relation for optimum coupling is used for both type of waveguide. These are : w/a $=0.64$ for circular corrugated waveguide with coupling coefficient of 0.98 and w/a $=0.3$ for smooth walled rectangular waveguide with coupling coefficient of 0.85 . There would be change in the optical components for these waveguide.

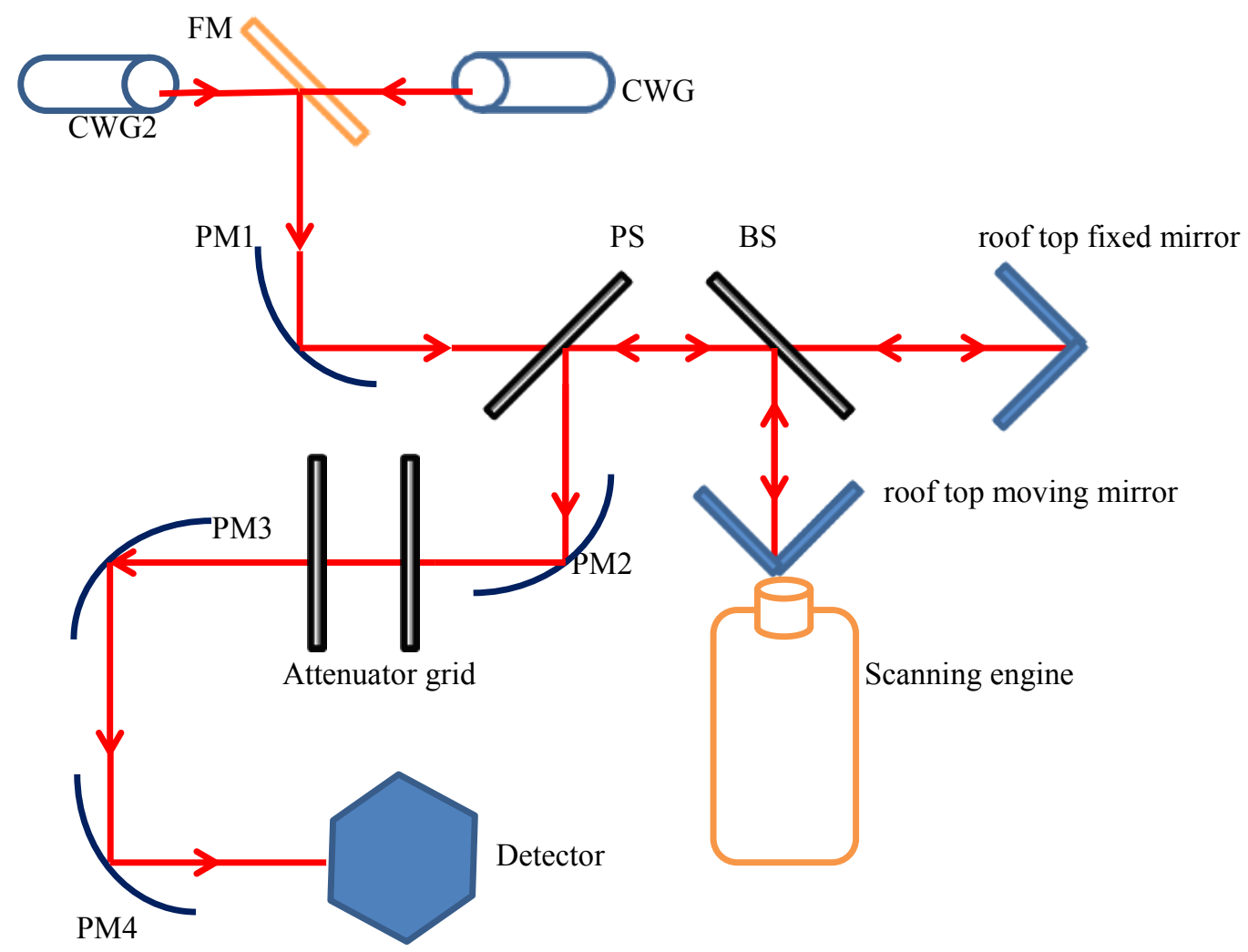

CWG circular waveguide, FM flat mirror

PM parabolic mirrors, PS polarizer selector, BS beam splitter

Fig. 2. Schematic lay out of connection optics \& Michelson interferometer optics 


\section{Allowable calibration error and integration time required for calibration}

For ECE electron temperature profile measurement, the total error should be $5 \%$ or less. The r.m.s. error should be $2.5 \%$ or less. While for power loss and spectral information, the r.m.s. error is also $2.5 \%$. For this, measurement from two views should be compared. Therefore, the r.m.s. error for each line of sight would need to be $<1.8 \% \mathrm{rms}$.

The statistical noise during plasma measurement is negligible for ITER ECE Michelson interferometer measurement. Therefore error in the calibration of the ECE system is dominated and should be less than $1.8 \%$. The calibration error depends on the error of the source temperature measurement, the error in knowledge of emissivity and statistical error in the calibration. In these, the accuracy in the surface temperature measurement is good $(<0.13 \%)$. The accuracy in the knowledge of the emissivity of the calibration source is proximately $\pm 1.2 \%$ or better [5]. Therefore; statistical error in calibration data should be $1.35 \%$ in r.m.s. to achieve total error of $1.8 \%$ r.m.s.

For $2 \%$ accuracy ( $1 \%$ r.m.s) the Signal to noise ration equals to 100 and the integration time $\mathrm{Ti}$ is given by[6]

$$
T_{i}=40000\left(\frac{N E P}{P_{S}}\right)^{2}
$$

$\mathrm{P}_{\mathrm{s}}$ is the calibration source power falling on the detector and can be expressed

$$
P_{s}=K \theta_{s} f^{2} \Delta f E_{t} T_{t l}
$$

Where $\theta_{\mathrm{s}}\left(=973{ }^{\circ} \mathrm{K}\right)$ is the source temperature, $\mathrm{f}$ is the frequency of radiation, $\Delta \mathrm{f}(=10 \mathrm{GHz})$ is frequency resolution of the interferometer, $E_{t}\left(=1.28 \times 10^{-5} \mathrm{~m}^{2} \mathrm{Sr}\right)$ is the etendue, $T_{t l}$ is the transmitivity from source to the detector and $\mathrm{K}=2.44 \times 10^{-41}$. We consider $\mathrm{NEP}=1 \times 10^{-12} \mathrm{~W} / \mathrm{Hz}^{-1 / 2}$.

The estimation of total attenuation on different transmission line is depicted in figure 3.The required integration time for the calibration is depicted in figure 4 for three types of waveguide transmission line. The integration time for smooth walled circular waveguide is reasonable and it is approximately 27 hours. In this one has to collect data every 2 hours with low accuracy and then take average data with long integration time for required calibration accuracy.

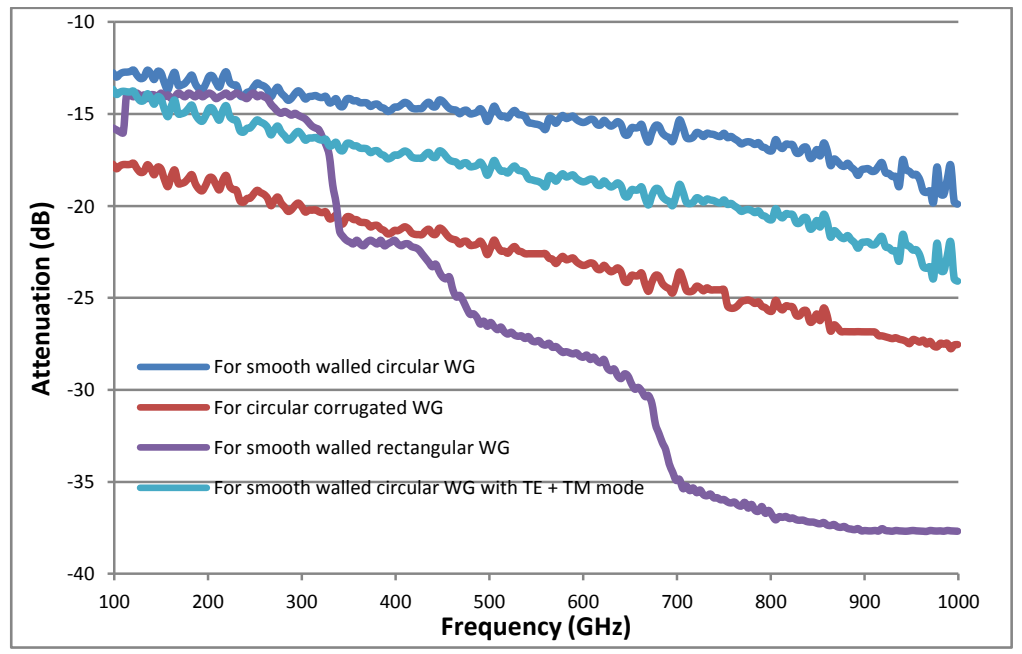

Fig. 3. Estimated total attenuation of transmission line of all three waveguides with three quartz windows, polarizer splitter box, 43 meter WG length, six miter bends and waveguide connection optics 


\section{Conclusions}

The conceptual design parameters of the O-mode radiometer would be accomplished the measurement requirement of the first harmonic O-mode radiation for ITER plasma. The required calibration time is fair for the proposed conceptual design of the Michelson interferometer and estimated attenuation of the transmission line.

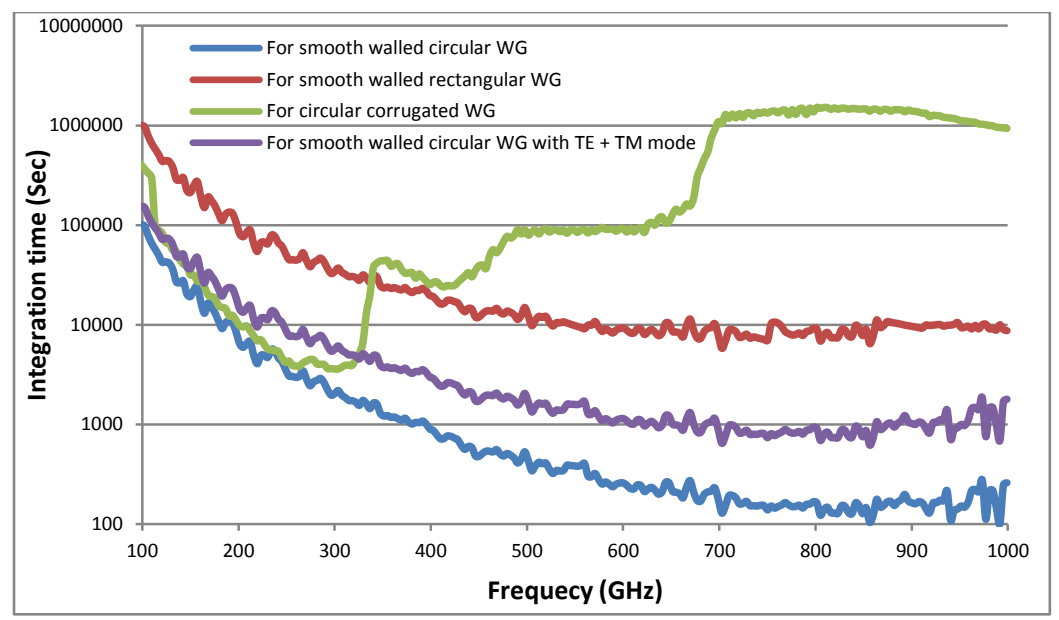

Fig. 4. Required integration time for calibration

\section{References}

1. M. Austin et al., these Proceedings

2. ECE system DDD, UID : 679HW9

3. P. Buratti and M. Zerbini, Rev. Sci. Instrum. 664208 (1995)

4. Paul F. Goldsmith, Quasioptical systems, IEEE Press/Champman \& Hall Publishers series on Microwave Technology and RF, PP169

5. Thomas C. Hsu, PhD Thesis on The Submillimeter wave ECE diagnostic for the Alcator-CMod Tokamak (1993)

6. Marco Zerbini, C. Sozzi, A. Simonetto, pp 208 in Proceeding of the $15^{\text {th }}$ Joint workshop on $E C E \& E C R H$, Yosemite National Park, California, USA, 10 - 13 March (2008) 